\title{
Mammary developmental fate and breast cancer risk
}

\author{
Daniel Medina
}

Baylor College of Medicine, One Baylor Plaza, Houston, Texas 77030, USA

(Requests for offprints should be addressed to D Medina; dmedina@bcm.tmc.edu)

\begin{abstract}
The ovarian hormones, estrogen and progesterone, play a pivotal role in normal and neoplastic development of the mammary gland. These hormones have a paradoxical role as long duration of estrogen and progesterone are associated with increased breast cancer risk, while short duration of pregnancy level doses are associated with a reduced breast cancer risk. The protective effects of estrogen and progesterone, as well as pregnancy, have been extensively studied in animal models. Recent studies have demonstrated that these hormones induce alterations in gene expression in the mammary epithelial cells which persist for a long time after the hormones are withdrawn from the host. It is postulated that hormones induce a switch in mammary developmental fate which decreases the risk of breast cancer over the lifetime of the host. Some of the possible cellular pathways persistently altered by short term hormone exposure are a decrease in growth factors and an increase in apoptosis. The expression of these genes, in turn, may be affected by alterations in genes regulating chromatin remodeling. The relative contributions of host-mediated factors and mammary cell intrinsic factors remain to be determined. The current studies have moved this research area from the biological to the molecular realm and offer the potential for directing prevention efforts at specific molecular targets.
\end{abstract}

Endocrine-Related Cancer (2005) 12 483-495

\section{Introduction}

Breast cancer is a major cancer of women in the United States and Western Europe with approximately 211000 new cases predicted in the United States in 2005 (American Cancer Society 2005). Breast cancer accounted for approximately $32 \%$ of new cancers diagnosed in US women in 2004 (American Cancer Society 2005). The rate of increase of breast cancer incidence slowed in the 1990s; at the same time, the incidence of in situ breast lesions, mainly ductal carcinoma in situ (DCIS), increased so that approximately 59000 new cases of in situ lesions occurred in 2004 (American Cancer Society 2005). The mortality rate was relatively constant through the last quarter of the 20th century before showing a significant decrease starting in the early 1990s. Early diagnosis and continuing new therapeutic approaches have managed to prevent the epidemic from causing a concomitant increase in death. Nevertheless, the death of 40000 women due to invasive breast cancer remains a sobering fact and indicates the need to understand this disease in greater depth and to develop new interventions, both preventive and therapeutic.

Current understanding of the central role of hormones in the genesis of breast cancer is based on over 100 years of studying breast cancer. Beatson in 1896 demonstrated that removing the source of steroid reproductive hormones by bilateral oopherectomy was an effective therapeutic treatment. The presence of estrogen receptor (ER) is the most widely used predictive factor of breast cancer response to treatment and was the basis for developing selective estrogen receptor modulators (SERMs; i.e. tamoxifen and raloxifene) and aromatase inhibitors (Jordan et al. 1991, Osborne 1998, Brodie et al. 1999, Goss 2004) as adjuvant therapy against breast cancer recurrence. Additionally, recent trials have documented that hormone replacement therapy that includes both estrogens and progestins imparts a greater breast cancer risk than estrogens alone (Santen 2003). 


\section{Epidemiological considerations}

Breast cancer incidence rates are influenced by age, genetics, reproductive history, radiation, socioeconomic status, place of residence, and ethnicity (McPherson et al. 2000). Reproductive history is the strongest and most consistent risk factor outside of genetic background and age (McPherson et al. 2000, Harris et al. 1992, Kelsey \& Gammon 1991, Althuis et al. 2004, Ursin et al. 2004). Early menarche, late menopause, parity, and late age of first pregnancy are each independent risk factors. It has been estimated that breast cancer risk is reduced $10-20 \%$ for each year of delay in the onset of menarche (Henderson et al. 1991, Harris et al. 1992). It is of interest that the high calorie Western-style diet pre-disposes children to an earlier puberty (Harris et al. 1992). Additionally, the phenomena of a Western-style diet, decreased age of menarche, and increased breast cancer incidence are now occurring in Japan and other countries in Asia. The early age of menarche translates into earlier hormone exposure (estrogen and progesterone) and breast epithelial cell growth (Henderson et al. 1991). Epidemiological studies have indicated that early age of menarche (i.e. 12.8 years vs $\geq 17$ years old) and height $\left(90^{\text {th }}\right.$ vs $10^{\text {th }}$ percentile) confer a 1.5 -fold and 1.3-fold increase in risk, respectively (Harris et al. 1992). A recent study shows that height and body mass index (BMI) at age 14 are associated with an increased risk in adult life (Ahlgren et al. 2004). These latter two measurements are considered to be surrogate markers for growth stimulatory activities in the prepubertal and adolescent years.

Ironically, although early menarche and total years of hormone exposure are risk factors for increased incidence, early age of first pregnancy ( $\geq 20$ years of age) is a strong protective factor. The protective factor is especially observed in postmenopausal women, the period of peak incidence. Parity-induced protection against breast cancer is principally dependent on the timing of the first full-term pregnancy but also is affected by total number of pregnancies (Albrektsen et al. 2005). The association is independent of the number of live births. Aborted pregnancies are not associated with decreased risk for breast cancer (Melbye et al. 1997, Beral et al. 2004). The protective effect of early first pregnancy has been repeatedly demonstrated in numerous epidemiological studies and provides a physiologically operative model to achieve practical and affordable prevention of breast cancer in humans (Henderson et al. 1991). The logic and rationale for understanding the molecular basis for hormone-mediated prevention of breast cancer are based on the consistent observations in human epidemiological studies and the strong confirmatory experiments in rodent breast cancer models.

\section{Protective effect of pregnancy}

There are many different ways to prevent carcinogenesis. The concept developed by Wattenberg (1997) emphasizes the distinction between blocking agents and suppressing agents. The former prevent carcinogenic chemicals from interacting with target molecules, whereas the latter prevent the development and/or expression of the transformed cells. With respect to the mammary gland, agents such as retinoic acid (Welsch 1987, Wu et al. 2002), selenium (Ip \& Medina 1987, Medina et al. 2001b), and D-limonene (Crowell et al. 1992) act as both blocking and suppressing agents, depending on the time intervals of application. It is more difficult to classify endogenous natural factors such as estrogen and progesterone. These agents do not fit the categories of suppressing or blocking agents since their effects can persist long after the withdrawal of the agents (Grubbs et al. 1985, Rajkumar et al. 2004). This consideration serves to emphasize the unique nature of hormonal prevention of methylnitrosourea (MNU)-induced carcinogenesis of the mammary gland. As illustrated in Table 1, prevention of mammary carcinogenesis can be attained by a single pregnancy or by hormone regimens comprising the two main hormones of pregnancy, estrogen and progesterone, or by human chorionic gonadotropin. Of the numerous experiments addressing this question, only the more pertinent experiments are summarized in Table 1.

There are two different experimental models that demonstrate parity/hormone-induced protection (Fig. 1). In the first model, one half of the animals undergo hormonal stimulation, the mammary gland is allowed to involute completely, and then the carcinogen is administered to the hormone-treated and AMV animals. This model is termed the 'pre-treatment model' and most of the experiments use this experimental protocol. In the second model, the animals are treated with a carcinogen, and then one half are exposed to hormone treatment for a specified time period. This model is referred to as the 'posttreatment' model. Unless otherwise stated, most comments refer to the pre-treatment model. The distinction is important as the underlying mechanisms for protection are likely to be different between the two models. 
Table 1 Prevention of Mammary Tumorigenesis by Parity and Hormones

\begin{tabular}{|c|c|c|c|c|}
\hline \multirow[b]{2}{*}{ Hormone treatment } & \multirow[b]{2}{*}{ Carcinogen (days) ${ }^{a}$} & \multicolumn{3}{|c|}{ Tumorigenesis (control/treated) } \\
\hline & & MC incidence ${ }^{b}$ & Inhibition (\%) & Ref. \\
\hline Pregnancy & $\operatorname{DMBA}(21 \mathrm{~d})^{\mathrm{c}}$ & $71 / 0$ & 100 & (Moon 1969) \\
\hline Pregnancy/lactation & DMBA (50 d) & $71 / 11$ & 84 & (Moon 1969) \\
\hline Pregnancy & DMBA (21 d) & $48 / 6$ & 88 & (Russo \& Russo 1980) \\
\hline Pregnancy & DMBA $(15 d)$ & $70 / 14$ & 80 & (Sinha et al. 1988) \\
\hline$E+P(20 \mu g / 4 m g)$ & MNU $(21 d)^{d}$ & $61 / 13$ & 79 & (Grubbs et al. 1985) \\
\hline$E+P(5 \mu \mathrm{g} / 2 \mathrm{mg})$ & MNU (21d) & $61 / 19$ & 69 & (Grubbs et al. 1988) \\
\hline hCG (10 lu) & DMBA $(21 d)$ & $44 / 18$ & 52 & (Russo IH et al. 1990) \\
\hline Pregnancy/lactation & DMBA $(100 \mathrm{~d})^{\mathrm{e}}$ & $38 / 16$ & 58 & (Russo IH et al. 1991) \\
\hline Pregnancy & DMBA $(130 d)^{e}$ & $38 / 18$ & 53 & (Russo IH et al. 1991) \\
\hline Pregnancy & MNU & $80 / 17$ & 79 & (Thordarson et al. 1995) \\
\hline$E+P(30 \mathrm{mg} / 30 \mathrm{mg})$ & MNU & $55 / 0$ & 100 & (Guzman et al. 1999) \\
\hline$E+P(20 \mu \mathrm{g} / 20 \mathrm{mg})$ & MNU (28d) & $56 / 10$ & 82 & (Sivaraman et al. 1998) \\
\hline Pregnancy/lactation $^{f}$ & MNU (168d) & $33 / 20$ & 40 & (Yang et al. 1999) \\
\hline Pregnancy/lactationg & MNU (75d) & $54 / 8$ & 85 & (Yang et al. 1999) \\
\hline Pregnancy $^{h}$ & MNU (75d) & $54 / 35$ & 35 & (Yang et al. 1999) \\
\hline Pregnancy ${ }^{i}$ & DMBA (15d) & $70 / 25$ & 64 & (Medina \& Smith 1999) \\
\hline
\end{tabular}

${ }^{a}$ Interval in days between end of E/P treatment, parturition (or weaning), and treatment with chemical carcinogen.

${ }^{\mathrm{b}} \mathrm{MC}=$ mammary carcinoma; $\mathrm{MC}$ incidence in control rats/MC incidence in treated rats.

${ }^{\mathrm{C}} \mathrm{DMBA}=7,12$-dimethyl benzanthracene

${ }^{\mathrm{d}} \mathrm{MNU}=$ methylnitrosourea

eRats were 180-190 days of age when administered carcinogen.

${ }^{f}$ Experienced one delivery of pups at 105 days of age (posttreatment model).

gExperienced one delivery of pups at 113 days of age (pretreatment model).

hExperienced one delivery of pups at 109 days of age without lactation (pretreatment model).

'All experiments used rats except this experiment, which used two different mouse strains.

\section{Effect of pregnancy, pregnancy and lactation}

Table 1 clearly demonstrates that pregnancy is an efficient physiological process to reduce mammary carcinogenesis. Most of the pregnancy experiments have been performed in outbred Sprague-Dawley rats, inbred Lewis and Wistar-Furth rats, and inbred mice. In most of the studies there is greater than $75 \%$ inhibition of the incidence of carcinomas. Pregnancy alone and pregnancy and lactation, occurring both prior to carcinogen administration and following carcinogen exposure, significantly reduce the incidence of mammary carcinomas and the number of palpable carcinomas per rat, and significantly prolong the latency. Epidemiological literature on the history of breast-feeding in relation to breast cancer risk in humans also shows that prolonged breast-feeding is additionally protective against breast cancer among premenopausal women (Althuis et al. 2004, PerezEscamilla \& Guerrero 2004). In rodents, the protective effect of pregnancy was observed even when the carcinogen was administered 100-130 days after the first parturition, indicating a long-lasting alteration in the state of sensitivity of the mammary gland. Thus, the rodent model represents an extensively validated model for the human paradigm.

In most experiments pregnancy was as effective as pregnancy and lactation ( $86 \%$ vs $83 \%$ inhibition respectively), so that the complete functional differentiation of the mammary gland would appear not to be necessary for protection from the carcinogenic insult. Pregnancy can be mimicked by estrogen and progesterone administration. It is known that estrogen can increase prolactin levels, so the role of prolactin has to be considered in any discussion of mechanisms. However, two studies using the NMU-rat model have shown that continuous treatment with perphenazine, a prolactin-elevating drug, does not lead to a protective state. The studies were performed in both the post-treatment model (Guzman et al. 1999) and the pre-treatment model (D Medina, unpublished observations). The length of pregnancy necessary to confer protection has yielded contradictory results. On the one hand, the experiments of Sinha et al. (1988) suggest that interrupted pregnancies of 5, 10, and 15 days resulted in $48 \%$ tumor incidence versus $79 \%$ tumor incidence for age-matched controls and 14\% tumor incidence for full-term pregnancy. In a different 
Pretreatment

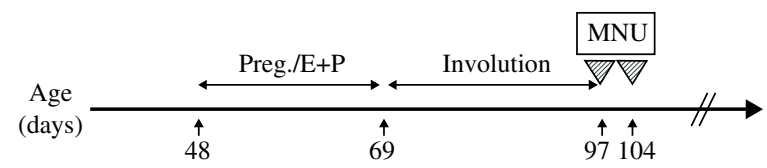

Posttreatment

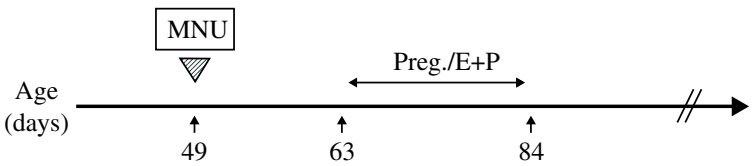

Figure 1 The two experimental treatment models for studying the effect of hormones on chemical carcinogen-induced mammary tumorigenesis in the rodent. The carcinogen, methylnitrosourea (MNU) is more commonly used in current rat studies, whereas 7,12-dimethylbenz $(\alpha)$ anthracene is used in mouse studies. Pregnancy or specific hormone treatment like estrogens $(E)$ or progestins $(P)$ occur prior to carcinogen (pre-treatment model) or after carcinogen administration (post-treatment model). In either model, a sufficient period of time is allowed between hormone and carcinogen exposure.

study (Russo et al. 1992), pregnancy interruption at day 12 did not confer any difference in protection compared with full-term pregnancy $(78 \%$ vs $71 \%$ respectively). There were only two basic differences in the experiments. Much larger group sizes were used (25-27 vs 7-9 rats respectively) in the Sinha experiment, and the interval between end of hormone stimulation and carcinogen treatment was 15 versus 21 days respectively. One can argue that either the larger group size or the longer time interval was the important variable. The larger group size $(>25$ animals per group) of the first study yields more powerful statistical data than the nine animals in one pregnancy-interrupted group. Additionally, the fact that only 5 days of hormone stimulation of pregnancy was sufficient to reduce the risk by $39 \%$ is startling. This result suggests that the initial act of hormone stimulation of mammary gland development induces sufficient changes in cell type and/or cellular regulatory pathways to create a significantly resistant cellular phenotype. After 5 days of stimulation, DNA synthesis is just beginning, morphological differentiation is minimal (Grubbs et al. 1988, Medina et al. 2001a), and subsequent epithelial cell involution is rapid. Therefore, it is unlikely that the resistance to carcinogen-induced transformation could be attributed to the presence of functionally differentiated lobuloalveolar cells. Furthermore, the perphenazine experiments referred to in the preceding paragraph indicate that lobuloalveolar differentiation per se, is not a critical or determining factor in resistance to carcinogen-induced tumorigenesis. It is more likely that hormones upregulate a specific regulatory pathway(s) which persist in the cells and progeny thereof.

Table 1 also shows that hormonal treatment to mimic pregnancy using either estrogen and progesterone or human chorionic gonadotropin is also effective in reducing mammary tumor incidence in rodents. Human chorionic gonadotropin causes a rise in endogenous sex steroids. Like pregnancy, hormone mimicry of pregnancy has been investigated in both the pre-treatment and post-treatment models of prevention. It is clear that both estrogen (E) and progesterone (P) are required to induce the maximum resistant phenotype; however, the use of single hormone treatments has yielded tantalizing results (Grubbs et al. 1985). Estrogen treatment $(20 \mu \mathrm{g} \mathrm{E} /$ day $\times 35$ days) did not significantly alter tumor incidence or the average number of tumors per rat. Progesterone treatment $(4 \mathrm{mg} /$ day $\times 35$ days $)$ delayed both the mean number of tumors per rat $(0.55$ vs $1.45, P<0.05)$ and the mean latency of tumor appearance (120 vs 108 days). However, the incidence of cancers was not significantly lower compared with animals administered hormone vehicle alone. A recent study by Medina et al. (2001a) shows that rats treated with either estrogen $(20 \mu \mathrm{g})$ or progesterone $(20 \mathrm{mg})$ alone for 21 days in a pre-treatment model was insufficient to confer protection. This result supports the earlier conclusion of Grubbs et al. (1988) that both hormones are necessary for the protective effect in a pretreatment model.

In contrast, the effects of $\mathrm{E}$ and $\mathrm{P}$ given singly were tested to determine the ability of these hormones to induce protection from mammary carcinogenesis in a post-treatment model (Rajkumar et al. 2001). It was shown that treatment with E2 alone, at a critical dose of $100 \mu \mathrm{g} / \mathrm{silastic}$ tubing, provided a pregnancy level of circulating E2 and a protection from mammary carcinogenesis compared with the AMV. In a recent study (Rajkumar et al. 2004), these same authors demonstrated that a one week exposure to $100 \mu \mathrm{g}$ ethynyl estradiol plus $30 \mathrm{mg}$ megesterol or norethindrone imparted a dramatic protective effect in the post-treatment model.

\section{Mouse model for hormone-induced protection}

Recently, we documented, in three different mouse strains, hormone-induced refractoriness of the involuted mouse mammary gland to chemical 
carcinogenesis (e.g., 7,12-dimethylbenzanthracene, DMBA) under conditions that are similar to those reported in rats and presumed to be operative in humans (Medina \& Smith 1999, Medina \& Kittrell 2003). Using the BD2fF1 strain of mice, we showed that mammary tumor incidence was statistically significantly reduced from 70 to $25 \%$ upon pregnancy and involution. In the $\mathrm{C} 3 \mathrm{H} / \mathrm{Sm}$ mice, mammary tumor incidence was decreased from 57 to $21 \%$, although the decrease did not reach statistical significance because of smaller group sizes. In BALB/c mice, mammary tumor incidence was decreased from $63 \%$ to $17 \%$, $(P<0.05)$. The histopathology of the mammary tumors arising in parous versus AMV mice was similar and included both type $\mathrm{B}$ adenocarcinomas and squamous adenocarcinomas. An earlier study had concluded that parous BALB/c mice were resistant to mammary carcinogenesis induced by MNU (Swanson et al. 1995). These results demonstrate that the mouse is a suitable model to examine the mechanism of hormone-induced refractoriness to carcinogen-induced mammary tumorigenesis. This is an important piece of information because it now allows the use of gene $\mathrm{KO}$ and transgenic mouse models to test specific hypotheses regarding the function of genes involved in the protection, e.g. p53 KO, cyclin D1 KO mice, PR KO mice, SRC3 KO mice, etc.

\section{Mechanisms of protection}

\section{Initiation vs promotion}

A consideration of the majority of the literature indicates that experiments have not specifically addressed the question of whether hormone-induced refractoriness is targeted to the initiation stage or promotion stage of carcinogenesis. However, there are several recent results stated in the literature that address this question (Yang et al. 1999, Reddy et al. 2002, Nandi et al. 2005). The results demonstrate that the hormone-induced refractory gland, upon autopsy contains small, nonpalpable microtumors in the absence of palpable tumors. The frequency of the microtumors was less than or equal to that found in MNU-treated AMV virgins. These experiments, performed in the pre- and post-treatment models, definitely demonstrate that pregnancy primarily delays progression. Along these lines, the provocative experiments by Thordarson et al. (2001), that continuing the same doses of hormones for an extended period of time (20 rather than 3 weeks) results in abundant tumor development equivalent to the $\mathrm{AMV}$, indicate that hormones can promote progression, if administered continuously. A similar conclusion was reached in the parous mouse using pituitary isografts as the source of hormones (Swanson et al. 1995).

\section{Induction of differentiation}

Studies by Russo \& Russo $(1987,1997)$ have led to the hypothesis that differentiation of the mammary gland, whether induced by pregnancy or hormones, results in the removal of the population of cancer-susceptible cells and hence confers protection against breast cancer. A gland that has encountered a full-term pregnancy has achieved a greater degree of terminal differentiation than that which is present in a similarly aged virgin gland. In this scenario, it has been postulated that the pregnancy-induced differentiation rids the gland of a specific population of susceptible cells (present in the terminal end buds (TEBs) and terminal ducts) which are considered targets of tumorigenesis (Russo \& Russo 1987, 1997). The highly mitotic terminal ducts are differentiated to less mitotic alveolar cells and progeny thereof.

The 'terminal differentiation' hypothesis was an attractive but oversimplified hypothesis that cannot explain the beneficial effects of hormones for several reasons. First, the appropriate comparison is between the AMV, where TEB are few, and the hormonetreated gland. Second, the process of pregnancy does not lead to persistent morphologically recognizable differentiated cells, i.e. persistent lobules. In rats, there is no consistent alteration in morphological differentiation between the AMV gland and the parous involuted gland when surveyed over different strains (Sinha et al. 1988, Thordarson et al. 1995). Hence, differentiation per se cannot explain the mechanisms of hormone-induced protection. Third, placental lactogen, which promotes differentiation, has no protective effect against breast cancer. Fourth, as discussed earlier, perphenazine (PPZ) is a dopamine receptor inhibitor that causes acute release of prolactin from the anterior pituitary of rats. This release of prolactin causes proliferation and differentiation of the mammary gland to a near-lactational state. Despite inducing morphological and functional differentiation of the mammary gland, PPZ is ineffective in offering protection from $\mathrm{MNU}$-induced carcinogenesis in rats in the pre- or post-treatment model (Guzman et al. 1999).

\section{Decreased proliferation activity}

There is strong evidence to suggest that decreased susceptibility of the gland to carcinogenesis with increasing age is correlated with a decrease in the 
highly proliferating cell populations of the mammary gland, i.e. the TEBs and terminal ducts. As a corollary to Russo's differentiation hypothesis, the differences in susceptibility to carcinogen-induced tumorigenesis between parous and age-matched virgin (AMV) glands could be explained by the differences in proliferation indices. This alternative explanation is unlikely. In 9-100-day old virgin rats, there are few TEBs and a very low cell proliferative index, yet the susceptibility is still very high (since $60-70 \%$ of the rats develop mammary adenocarcinomas within a short-time period after MNU). It has been reported that there are no differences in the overall cellular kinetics between parous rats and AMVs (Sinha et al. 1988, Sivaraman et al. 1998).

\section{Altered hormonal milieu}

Another hypothesis emphasizes that resistance to mammary tumorigenesis in parous rats may be caused by persistent changes in the parous rat mammary gland hormonal environment resulting in permanent biochemical alterations in the mammary epithelia (Musey et al. 1987, Thordarson et al. 1995). Circulating levels of prolactin appeared lower (although this was not statistically significant) in the parous rat compared with the AMV (Thordarson et al. 1995). Growth hormone $(\mathrm{GH})$ concentration was significantly reduced in parous rats as compared with AMV rats (Thordarson et al. 1995). The parous animals also had decreased levels of ER and EGF-R (Thordarson et al. 1995). The reduction in the circulating levels of GH caused a reduced susceptibility of the parous rats to mammary carcinogenesis, possibly by decreasing the levels of ER, EGF-R and/or IGF-1 in the mammary gland. The role of the GH-IGF1 axis in hormoneinduced protection is supported by two additional results (Thordarson et al. 2004a,b). First, NMUinduced mammary tumorigenesis is almost totally absent in GH-deficient spontaneous dwarf rats (SDR). SDR rats given GH or IGF-1 before and after NMU-treatment exhibited a normal $(\mathrm{GH})$ or near normal (IGF-1) tumorigenic response. Estradiol (E2) and progesterone $(\mathrm{P} 4)$ treatment did not restore tumorigenesis. Furthermore, E2 + P4 blocked the response to GH. SDR rats have a normal circulating concentration of prolactin (PRL), indicating PRL was not a factor in the low tumorigenic incidence (Thordarson et al. 2004a). Second, IGF-1 treatment of parous rats for 60 days after NMU-treatment increased the mammary tumor incidence compared with untreated parous rats and to AMV rats (Thordarson et al. 2004b).
In some studies, the results show that pregnancy reduces the number of ER and/or PR positive cells in the normal mammary glands of the parous rat (Thordarson et al. 1995, Yang et al. 1999). An inadequate hormonal environment could contribute to low ER and PR levels, low levels of proliferation, and slow rate of progression. This hypothesis was developed in the post-treatment model and has not been tested in the pre-treatment model. In addition, parts of the hypothesis, i.e. the altered frequency of ER positive cells in parous rats, have not been reproduced in other rat strains (Sivaraman et al. 2001b). In addition, it is important to examine whether the stroma of parous animals supports proliferation of mammary epithelial cells in a quantitatively different manner than the stroma of AMVs. It has been demonstrated that the reproductive state does persistently alter mammary stroma extracellular matrix composition and function (Schedin et al. 2004).

\section{Cell fate hypothesis}

The cell fate hypothesis states that in the mammary gland of the immediate postpubescent female, hormones induce a molecular switch in stem cells that results in cells with persistent changes in the intracellular regulatory loops governing proliferation and response to DNA damage (Sivaraman et al. 1998). In this 'cell fate hypothesis' at a critical period in adolescence, the hormonal milieu of pregnancy affects the developmental fate of a subset of mammary epithelial cells. The consequence of hormone exposure is the induction of persistent differences in signal transduction pathways, growth factors, cell cycle regulators, transcription factors, and/or other unknown regulatory molecules between the epithelial cells in the hormone-treated and the mature virgin mammary gland. These molecular changes are manifested in at least three cellular responses that differentiate the two cell states: first, a block in carcinogen-induced proliferation (Sivaraman et al. 1998); second, a block in proliferation of estrogenreceptor positive cells (Sivaraman et al. 2001b); and third, the appearance of a new cell type, termed parityinduced mammary epithelial cells (PI-MEC) (Wagner et al. 2002).

First, the number of apoptotic cells is low and comparable in the hormone-exposed and AMV glands at the time of carcinogen challenge, and remains low 8 days after MNU. The number of BrdU-labeled cells at the time of carcinogen challenge is low in both the AMV (1.8\%) and hormone-exposed $(0.8 \%)$ animals (Sivaraman et al. 1998). On the eighth day after MNU 
treatment, cell proliferation in the AMV (5.7\%) was significantly different from the parous involuted $(1.2 \%)$ and $\mathrm{E} / \mathrm{P}$-treated animals $(1.5 \%)$. This same relationship was seen in DMBA-treated BALB/c mice (Sivaraman et al. 2001a). Therefore, it appears that hormone treatment results in persistent alterations in intracellular pathways governing proliferation responses to carcinogens. The difference in proliferation frequency disappears by 20 days after carcinogen challenge. There are likely other cellular responses that are different between the two cell states, but these have not been elucidated yet.

Second, ER+ and PR + proliferating cells are rare in the developing mammary gland of a young virgin rat, and the two markers, receptor expression and proliferation, are dissociated from each other (Russo et al. 1999). This result is in accord with the current hypothesis that steroid receptor dependent regulation of proliferation is paracrine in the normal developing mammary gland (Anderson et al. 1998). The percentage of dual-labeled cells significantly increased in the mammary gland of a mature virgin rat (96-day old rat), and the two markers were positively associated. However, prior exposure of the mammary gland to pregnancy levels of $\mathrm{E} / \mathrm{P}$ results in the failure of the mammary gland to attain a positive association between steroid receptor expression and proliferation (Sivaraman et al. 2001b). These results have two important implications. The co-expression of steroid receptors and proliferation may be an early manifestation of an important molecular change in steroid receptor-dependent regulation of proliferation that increases susceptibility to tumor formation. As well, the ability to block association between the two markers might be one way in which pregnancy induces protection against breast cancer.

Third, the altered cell fate hypothesis is supported by recent studies demonstrating that pregnancy induces the appearance of a stable cell subpopulation that persists after involution (Wagner et al. 2002). The authors termed these cells parity-induced mammary epithelial cells (PI-MEC) and demonstrated that these cells provide the cells generated in subsequent pregnancies. Transplantation experiments demonstrate that these cells possess progenitor capabilities as they produced both luminal and myoepithelial cells. These PI-MEC are pluripotent and self-renewing (Boulanger et al. 2005). Interestingly, G.B7, one of the genes persistently elevated by pregnancy, colocalizes in the same tissue compartment occupied by the parityinduced cells (Ginger et al. 2001).

\section{Molecular studies}

\section{Gene Expression}

Several different groups have published extensive gene expression profiles of parous versus AMV mammary epithelial cells in rodent models. The results are summarized in Table 2. Ginger et al. (2001) used suppression subtractive hybridization (SSH) and identified over 200 genes that were persistently upregulated in the hormone-treated involuted glands of WistarFurth female rats. One gene, RbAp46, functions in chromatin remodeling. The other gene of interest is designated G.B7 and is a noncoding RNA. The importance of the upregulation of these unique genes is that they suggest epigenetic mechanisms for the persistent changes found in the hormone-treated mammary epithelial cells and for the altered cell fate. A similar mechanism may operate with hCG-induced protection. In this model, the effect of hCG is correlated with increased expression of inhibin and downstream activation of c-myc and other genes (Jiang et al. 2002). The authors show that the transcription of these genes is accompanied by increased acetylation of histones in a non-tumorigenic human breast cell line MCF-10F when treated with either hCG or inhibin.

In an elegant and comprehensive study by D'Cruz et al. (2002), the authors used DNA microarray technology to identify differentially expressed genes common in the parous involuted glands in multiple strains of both rats and mice. The authors identified 38 differentially expressed genes common to both species

Table 2 Evidence for altered gene expression in hormone-treated mammary gland

\begin{tabular}{|c|c|c|c|}
\hline Gene(s) & Method & Result & Reference \\
\hline Growth factors, TGF $\beta_{3}$ & DNA microarrays & $\uparrow \downarrow$ in parous gland & (D’Cruz et al. 2002) \\
\hline G.B7, RbAp46 (chromatin remodeling) & $\begin{array}{l}\text { Suppression subtractive } \\
\text { hybridization }\end{array}$ & $\uparrow$ in parous gland & (Ginger et al. 2001) \\
\hline Growth factors, pro-apoptotic & Agitent DNA microarray & $\uparrow \downarrow$ in E2 exposed gland & (Rajkumar et al. 2005) \\
\hline Pro-apoptotic & Northern blots & $\uparrow$ in hCG treated gland & (Srivastava et al. 1997) \\
\hline p53 & Immunohistochemistry & $\uparrow$ in parous gland & (Sivaraman et al. 1998) \\
\hline
\end{tabular}


that defined the parous involuted gland. The genes included increased expression of TGF $\beta 3$ and several of its downstream targets. The result of this upregulation would be inhibitory for cell growth. Additionally, there was decreased expression of several growth factors that included IGF-1 and amphiregulin. The result of the decreased expression of these genes would be a lowered stimulus for proliferation. The net result is a low, steady state for growth. One can speculate that responses to specific stimuli for proliferation might also be diminished. In fact, the response to chemical carcinogen-induced proliferation is reduced in the parous involuted glands of both rats and mice (Sivaraman et al. 1998, 2001a). Also, one has to consider that some of these genes (e.g. IFG1) might be downregulated due to decreased pituitary growth hormone as demonstrated by Swanson et al. (1995), Thordarson et al. (2004b).

In a third study using the post-treatment model in the rat, Rajkumar et al. (2005) using Agilent rat cDNA microarrays, identified a gene signature conferred by a protective dose of $200 \mu \mathrm{g}$ E2. Totally 20 genes were differentially expressed between the hormone treated and untreated groups. The genes included both proapoptotic (upregulated) and cell cycle regulatory (downregulated) genes.

Alterations in other genes have been shown by a variety of methods. In the post-treatment model where hCG induces a protective state, enhanced apoptosis is present during 40 days of treatment, but declines upon cessation of treatment. Northern blot studies indicate that mRNA of interleukin-1-B-converting enzyme, testosterone repressed prostate message 2, p53, c-myc and bcl-XS were upregulated and correlated nicely with the enhanced apoptotic activity in the gland (Srivastava et al. 1997). The expression of these genes reached a peak at 40 days of treatment and was still elevated 20 days after cessation of treatment. Some of these genes, such as bcl-XS, p53, c-myc and inlibin were also found in a cDNA microarray analysis (Russo et al. 2005). As in the initial Northern blot study, these genes reached the highest peak at 21 days, but were decreasing by 42 days post hCG treatment. However, there was a small set of genes that were persistently differentially expressed at 42 days of hCG treatment that the authors suggest confer a genetic signature for hCG treatment (Russo et al. 2005).

A common element that appears in these various studies is the downregulation of growth regulatory genes in the protected state, particularly in the pretreatment models studied by Ginger et al. (2001) and D'Cruz et al. (2002). In the post-treatment model, there is the additional element of pro-apoptotic genes that are upregulated (Rajkumar et al. 2005, Russo et al. 2005). The molecular studies support the observations at the cellular level where it was concluded that downregulation of the proliferative response was prevalent in the pre-treatment model (Sivaraman et al. 1998), whereas upregulation of apoptosis was observed only in the post-treatment model (Srivastava et al. 1997, Sivaraman et al. 1998).

\section{p53}

A striking cellular alteration present in the parous involuted glands in the pre-treatment model is the block in carcinogen-induced proliferation (Sivaraman et al. 1998). Alterations in apoptosis were not detected. This block is detected in both Wistar-Lewis rats and $\mathrm{BALB} / \mathrm{c}$ mice. Concomitantly with this block in proliferation is a significant increase in the level and nuclear localization of the tumor suppressor protein, $\mathrm{p} 53$, in the parous involuted gland. The increase occurs as early as 3 days after hormone treatment and is persistent until 28 days of involution and for 3 days after NMU treatment (Sivaraman et al. 1998, 2001a). Kuperwasser et al. (2000) have previously discovered that wildtype $\mathrm{p} 53$ is inactive in the virgin gland, but is functionally activated by hormone-exposure. The molecular mechanisms of this activation have not been elucidated yet, but the demonstration of hormonal activation of $\mathrm{p} 53$ supports a hypothesis for the role of p53 in the hormone-induced protective state. Additionally, it has been demonstrated that $\mathrm{p} 53$ functions in the DNA repair of polycyclic aromatic hydrocarbon adducts (Lloyd \& Hanawalt 2000).

The importance of p53 for the hormone-induced protective state has been directly tested using the p53null BALB/c mouse mammary epithelial cell model. The experiments demonstrated that the absence of p53 gene function abrogated the protective effect of hormones against carcinogen (DMBA)-induced mammary carcinogenesis (Medina \& Kittrell 2003). To date, this study represents the first and only identification of a specific gene product that mediates the protective effect of hormones. However, the development of several new and elegant conditional transgenic mouse models for c-myc, wnt, Brca-1 and cneu offer the promise of similar experiments (Deng 2002, Gunther et al. 2002, Moody et al. 2002, Gunther et al. 2003, Boxer et al. 2004, Chodosh 2005). Additionally, the identification of new candidate genes by microarray studies suggests that numerous genes can be tested for their roles in the hormoneinduced protective state using transgenic mouse models. 


\section{Beyond the current paradigms}

There are several important questions that need to be examined before we have a fuller understanding of the mechanisms and translatability of the hormoneinduced protective state. First, a critical testing is needed of the obligatory role of the genes and regulatory pathways involved in protection. Second, a dissection is required of the relative roles of the molecular changes intrinsic to the target cells for neoplastic transformation, i.e. mammary epithelium versus alterations in the pituitary gland and in the mammary stroma that affect progression of initiated mammary cells. Third, is the paradigm that has been developed using chemical carcinogen treated rats and mice applicable to mammary tumor models that are independent of chemical carcinogen initiation? This latter question is of interest for two reasons. First, the only experiment in the published literature using irradiation as an initiating agent for mammary tumorigenesis in the rat failed to demonstrate a hormone-induced protective effect (Holtzman et al. 1982). Second, polycyclic aromatic hydrocarbons, like DMBA, and nitrosoureas have not been demonstrated to be carcinogenic agents for the human breast.

The first two issues are being addressed by current research but it will be some years yet before there is a clear understanding of these issues. The third issue has been recently examined in the p53 null mammary epithelial model. The absence of $\mathrm{p} 53$ protein function confers an increased risk for breast tumorigenesis (Jerry et al. 2000). This is accompanied by chromosomal instability (Goepfert et al. 2000, Pati et al. 2004) and as yet, undefined secondary alterations in gene function. The model allows a test for the issue discussed above. If $\mathrm{BALB} / \mathrm{c}$ wildtype mice bearing p53-null normal mammary epithelial cells are exposed to E plus $\mathrm{P}$ for only 2 weeks during the duct elongation phase at 5 to 7 weeks of host age, (i.e. akin to immediate post-pubertal growth phase), then tumorigenesis is reduced over $90 \%$ over an ensuing 40 weeks (D Medina, unpublished observations). Interestingly, the inhibition of tumorigenesis is accompanied by a decreased steady state proliferation rate. These results need to be repeated in other models (e.g. c-myc, c-neu) in order to document conclusively the importance and relevance of the hormone-induced protective state as a prevention modality.

The cell fate hypothesis and the demonstration that both chemical carcinogen and oncogene models are subject to hormone-mediated protection raises a more fundamental question. Is there a unique developmental window that is particularly sensitive to hormone-mediated prevention of mammary cancer? For instance, would hormones induce protection in the mature virgin gland, say at 20-26 weeks of age? It is known in the pre-treatment model that hormones given at 7 to 10 weeks of age induce a protective state against DMBA-induced tumorigenesis whether DMBA is given at 14 weeks or 26 weeks of age (Moon 1969). The question is whether hormones affect the developing gland (4-12 weeks of age) differently than the mature gland ( $\geq 20$ weeks of age). There is considerable evidence in the literature that events occurring in the mammary gland during the development phase confer the greatest relative risk for tumorigenesis (Colditz \& Frazier 1995). For instance, for human breast cancer, the greatest risk in individuals exposed to a single large dose of radiation (i.e. Hiroshima and Nagasaki atomic bomb survivors) occurred in females $\leq 20$ years of age (Tokunaga et al. 1994, Land et al. 2003). The protective effects of a soy-based diet depend on soy intake during the adolescent years ( $\mathrm{Wu}$ et al. 2002). Finally, the recent demonstration that the highest quintile of height and BMI at age 14 is associated with subsequent breast cancer risk suggests the importance of growth stimuli during the peri-pubertal years (Ahlgren et al. 2004). These considerations are critical for understanding the importance and relevance of the cell fate hypothesis discussed earlier. A corollary to the hypothesis is that the developing gland (immediately post-pubertal) represents a unique window for altering cell fate and subsequent response to oncogenic initiating stimuli. If validated, then the optimum period for long term prevention modality would be in these years. A short time period (months) of exposure to non-invasive, normal cycling hormones would induce a long lasting protective effect. This hypothesis can be tested thoroughly in current experimental models and within 2 years, the validity of the hypothesis can be proven or disproven.

\section{Conclusion}

The area of hormone-induced protection of mammary cancer has been studied for over 30 years. The current studies have moved the area from the biological to the molecular realm. The central hypothesis has been redefined from a vague 'promoting differentiation' to a molecular-based 'altered cell fate.' It is likely that systemic and even stromal alterations also affect the fate of altered mammary epithelial cells. In its current form, the hypothesis states that upon exposure to hormones, the mammary epithelial cells comprising the young virgin gland undergo a series of changes in gene 


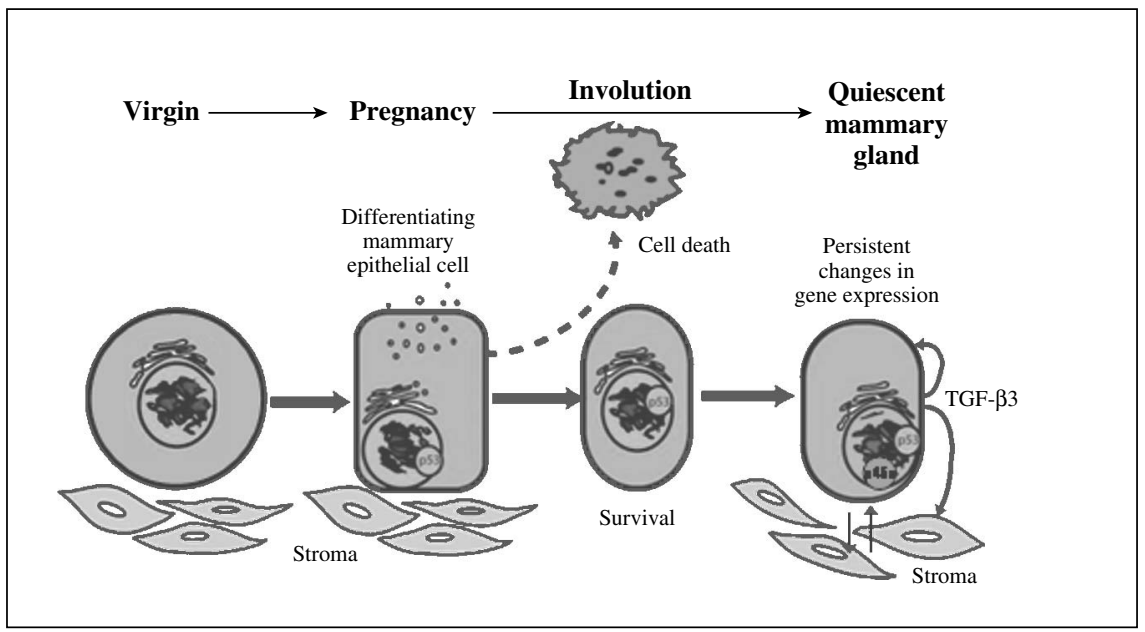

Figure 2 The 'Cell fate' hypothesis. In the mammary gland of the immediate postpubescent female, hormones stimulate the proliferation of a specific stem cell population (PI-MEC) which is accompanied by a molecular switch in these cells that results in persistent changes in intracellular regulatory loops governing proliferation and response to DNA damage. The hormonal milieu of pregnancy affects the developmental fate of a subset of mammary epithelial cells such that they become resistant to neoplastic transformation (either initiation and/or promotion). (Modified from Ginger \& Rosen 2003.)

expression affecting specific regulatory pathways resulting in a cell population of the parous involuted gland that is distinct from that of the age matched virgin (Fig. 2). In effect, the mammary epithelial cell has attained its complete developmental fate; a unique situation compared with most organogenesis where development is completed in the fetal stage or early postnatally. The consequences for prevention of human breast cancer are potentially enormous because they provide the basis for a non-invasive strategy using normal, endogenous hormones. The challenge will be to translate the basic concepts developed in the animal models to the human population where the affected individual is of young age and non-cancer bearing. However, there are very high risk human populations of young age where these prevention modalities could be justified.

\section{Acknowledgements}

The original results discussed here on the Wistar-Furth rat and BALB/c mouse models were supported by NCI P01 CA064255. I am grateful for the collaboration and interactions of the investigators on this program project, Bert W O'Malley, Orla Conneely, Jeffrey Rosen, Bill R Brinkley, Lakshmi Sivaraman, Melanie Ginger and Thea Goepfert. I greatly appreciate the technical assistance of Frances Kittrell, Anne Shepard, David Edwards and Jason Gay in the many rat and mouse experiments discussed herein, and the secretarial skills of Kathy Key. Without these dedicated and skillful individuals, the many results reviewed herein would not have been possible.

\section{References}

Ahlgren M, Melbye M, Wohlfahrt J \& Sorensen TL 2004 Growth patterns and the risk of breast cancer in women. The New England Journal of Medicine 351 1619-1626.

Albrektsen G, Heuch I, Hansen S \& Kvale G 2005 Breast cancer risk by age at birth, time since birth and time intervals between births: exploring interaction effects. British Journal of Cancer 92 167-175.

Althuis MD, Fergenbaum JH, Garcia-Closas M, Brinton LA, Madigan MP \& Sherman ME 2004 Etiology of hormone receptor-defined breast cancer: a systematic review of the literature. Cancer Epidemiology, Biomarkers and Prevention 13 1558-1568.

American Cancer Society. Facts and Figures (2005).

Anderson E, Clarke RB \& Howell A 1998 Estrogen responsiveness and control of normal human breast proliferation. Journal of Mammary Gland Biology and Neoplasia 3 23-36.

Beatson, GT 1896 On the treatment of inoperable cases of carcinoma of the mamma: suggestions for a new method of treatment with illustrative cases. Lancet 2 104-107.

Beral V, Bull D, Doll R, Peto R \& Reeves G 2004 Breast cancer and abortion: collaborative reanalysis of data from 53 epidemiological studies, including 83000 women with breast cancer from 16 countries. Lancet 363 1007-1016.

Boulanger CA, Wagner KU \& Smith GH 2005 Parityinduced mouse mammary epithelial cells are pluripotent, self-renewing and sensitive to TGF-betal expression. Oncogene 20 552-560. 
Boxer RB, Jang JW, Sintasath L \& Chodosh LA 2004 Lack of sustained regression of c-MYC-induced mammary adenocarcinomas following brief or prolonged MYC inactivation. Cancer Cell 6 577-586.

Brodie A, Lu Q, Liu Y \& Long B 1999 Aromatase inhibitors and their antitumor effects in model systems. Endocrine Related Cancer 6 205-210.

Chodosh LA 2005 Modeling human cancers in mice. Cancer Biology and Therapy 4 255-256.

Colditz GA \& Frazier AL 1995 Models of breast cancer show that risk is set by events of early life: prevention efforts must shift focus. Cancer Epidemiology, Biomarkers and prevention 4 567-571.

Crowell PL, Kennan WS, Haag JD, Ahmad S, Vedejs E \& Gould MN 1992 Chemoprevention of mammary carcinogenesis by hydroxylated derivatives of $d$-limonene. Carcinogenesis 13 1261-1264.

D'Cruz CM, Moody SE, Master SR, Hartman JL, Keiper EA, Imielinski MB, Cox JD, Wang JY, Ha SI, Keister BA \& Chodosh LA 2002 Persistent parity-induced changes in growth factors, TGF-beta3, and differentiation in the rodent mammary gland. Molecular Endocrinology 16 2034-2051.

Deng CX 2002 Tumor formation in Brcal conditional mutant mice. Environmental and Molecular Mutagenesis 39 171-177.

Ginger MR \& Rosen JM 2003 Pregnancy-induced changes in cell-fate in the mammary gland. Breast Cancer Research 5 192-197.

Ginger MR, Gonzalez-Rimbau MF, Gay JP \& Rosen JM 2001 Persistent changes in gene expression induced by estrogen and progesterone in the rat mammary gland. Molecular Endocrinology 15 1993-2009.

Goepfert TM, McCarthy M, Kittrell FS, Stephens C, Ullrich RL, Brinkley BR \& Medina D 2000 Progesterone facilitates chromosome instability (aneuploidy) in p53 null normal mammary epithelial cells. FASEB Journal 14 2221-2229.

Goss PE 2004 Changing clinical practice: extending the benefits of adjuvant endocrine therapy in breast cancer. Seminars in Oncology 31 S15-S22.

Grubbs CJ, Farnell DR, Hill DL \& McDonough KC 1985 Chemoprevention of $N$-nitroso- $N$-methylurea-induced mammary cancers by pre-treatment with 17 beta-estradiol and progesterone. Journal of the National Cancer Institute 74 927-931.

Grubbs CJ, Juliana MM, \& Whitaker LM 1988 Short-term hormone treatment as a chemopreventive method against mammary cancer initiation in rats. Anticancer Research 8 113-117.

Gunther EJ, Belka GK, Wertheim GB, Wang J, Hartman JL, Boxer RB \& Chodosh LA 2002 A novel doxycyclineinducible system for the transgenic analysis of mammary gland biology. FASEB Journal 16 283-292.

Gunther EJ, Moody SE, Belka GK, Hahn KT, Innocent N, Dugan KD, Cardiff RD \& Chodosh LA 2003 Impact of p53 loss on reversal and recurrence of conditional
Wnt-induced tumorigenesis. Genes and Development 17 488-501.

Guzman RC, Yang J, Rajkumar L, Thordarson G, Chen X \& Nandi S 1999 Hormonal prevention of breast cancer: mimicking the protective effect of pregnancy. PNAS 96 2520-2525.

Harris JR, Lippman ME, Veronesi V \& Willett W 1992 Breast cancer (1). The New England Journal of Medicine 327 319-328.

Henderson BE, Ross RK \& Pike MC 1991 Toward the primary prevention of cancer. Science 254 1131-1138.

Holtzman S, Stone JP \& Shellabarger CJ 1982 Radiationinduced mammary carcinogenesis in virgin, pregnant, lactating, and postlactating rats. Cancer Research $\mathbf{4 2}$ 50-53.

Ip C \& Medina D 1987 Current concepts on selenium and mammary tumorigenesis. In Cell and Molecular Biology of Experimental Breast Cancer, pp 479-494. Eds D Medina, GH Hepper, WR Kidwell \& E Anderson. New York: Plenum Press.

Jerry DJ, Kittrell FS, Kuperwasser C, Laucirica R, Dickinson ES, Bonilla PJ, Butel JS \& Medina D 2000 A mammary-specific model demonstrates the role of the p53 tumor suppressor gene in tumor development. Oncogene 19 1052-1058.

Jiang X, Russo IH \& Russo J 2002 Human chorionic gonadotropin and inhibin induce histone acetylation in human breast epithelial cells. International Journal of Oncology 20 77-79.

Jordan VC, Labadidi MK \& Langan-Fahey S 1991 Suppression of mouse mammary tumorigenesis by long-term tamoxifen therapy. Journal of the National Cancer Institute 83 492-496.

Kelsey JL \& Gammon MD 1991 The epidemiology of breast cancer. CA: A Cancer Journal for Clinicians 41 146-165.

Kuperwasser C, Pinkas J, Hurlbut GD, Naber SP \& Jerry DJ 2000 Cytoplasmic sequestration and functional repression of p53 in the mammary epithelium is reversed by hormonal treatment. Cancer Research 60 2723-2729.

Land CE, Tokunaga M, Koyama K, Soda M, Preston DL, Nishimori I \& Tokuoka S 2003 Incidence of female breast cancer among atomic bomb survivors, Hiroshima and Nagasaki 1950-1990. Radiation Research 160 707-717.

Lloyd DR \& Hanawalt PC 2000 p53-dependent global genomic repair of benzo[a]pyrene-7,8-diol-9,10-epoxide adducts in human cells. Cancer Research 60 517-521.

McPherson K, Steel CM \& Dixon JM 2000 ABC of breast diseases. Breast cancer-epidemiology, risk factors, and genetics. British Journal of Cancer 321 624-628.

Medina D \& Smith GH 1999 Chemical carcinogen-induced tumorigenesis in parous, involuted mouse mammary glands. Journal of the National Cancer Institute 91 967-969.

Medina D \& Kittrell FS 2003 p53 function is required for hormone-mediated protection of mouse mammary tumorigenesis. Cancer Research 63 6140-6143. 
Medina D, Peterson LE, Moraes R \& Gay J $2001 a$ Short-term exposure to estrogen and progesterone induces partial protection against NMU-induced mammary tumorigenesis in Wistar-Furth rats. Cancer Letters 169 1-6.

Medina D, Thompson H, Ganther H \& Ip C $2001 b$ Se-methylselenocysteine: a new compound for chemoprevention of breast cancer. Nutrition and Cancer $4012-17$.

Melbye M, Wohlfahrt J, Olsen JH, Frisch M, Westergaard T, Helwig-Larsen K \& Andersen PK 1997 Induced abortion and the risk of breast cancer. The New England Journal of Medicine 336 81-85.

Moody SE, Sarkisian CJ, Hahn KT, Gunther EJ, Pickup S, Dugan KD, Innocent N, Cardiff RD, Schnall MD \& Chodosh LA 2002 Conditional activation of Neu in the mammary epithelium of transgenic mice results in reversible pulmonary metastasis. Cancer Cell 2 451-461.

Moon RC 1969 Relationship between previous reproductive history and chemically induced mammary cancer in rats. International Journal of Cancer 4 312-317.

Musey VC, Collins DC, Musey PI, Martino-Saltzman D \& Preedy JR 1987 Long-term effect of a first pregnancy on the secretion of prolactin. The New England Journal of Medicine 316 229-234.

Nandi S, Guzman RC, Thordarson G \& Rajkumar L 2005 Estrogen can prevent breast cancer by mimicking the protective effect of pregnancy. In Hormonal Carcinogenesis IV, pp 153-165. Eds JJ Li, SA Li \& A Llombart-Bosch. New York: Springer.

Osborne CK 1998 Tamoxifen in the treatment of breast cancer. The New England Journal of Medicine 339 1609-1618.

Pati D, Haddad BR, Haegele A, Thompson H, Kittrell FS, Shepard A, Montagna C, Zhang N, Ge G, Otta SK et al. 2004 Hormone-induced chromosomal instability in p53 null mammary epithelium. Cancer Research 64 5608-5616.

Perez-Escamilla R \& Guerrero ML 2004 Epidemiology of breastfeeding: advances and multidisciplinary applications. Advances in Experimental Medicine and Biology 554 45-59.

Rajkumar L, Guzman RC, Yang J, Thordarson G, Talamantes F \& Nandi S 2001 Short-term exposure to pregnancy levels of estrogen prevents mammary carcinogenesis. PNAS 98 11755-11759.

Rajkumar L, Guzman RC, Yang J, Thordarson G, Talamantes F \& Nandi S 2004 Prevention of mammary carcinogenesis by short-term estrogen and progestin treatments. Breast Cancer Research 6 R31-R37.

Rajkumar L, Dang D-N, Hartnett MD, Hirschberg DL, Loh KC, Guzman RC, Thordarson G \& Nandi S 2005 Microarray analysis of estrogen-induced protection against breast cancer. In Hormonal Carcinogenesis $I V$, pp 420-425. Eds JJ Li, SA Li \& A Llombart-Bosch. New York: Springer.
Reddy M, Nguyen S, Farjamrad F, Laxminarayan S, Lakshmanaswamy R, Guzman R, Yang J \& Nandi S 2002 Short-term hormone treatment with pregnancy levels of estradiol prevents mammary carcinogenesis by preventing promotion of carcinogen initiated cells. Proceedings of the American Association for Cancer Research Annual Meeting 431964.

Russo J \& Russo IH 1980 Susceptibility of the mammary gland to carcinogenesis. American Journal of Pathology 100 497-512.

Russo J \& Russo IH 1987 Biological and molecular bases of mammary carcinogenesis. Laboratory Investigation $\mathbf{5 7}$ $112-137$.

Russo J \& Russo IH 1997 Role of differentiation in the pathogenesis and prevention of breast cancer. Endocrine-Related Cancer 4 7-12.

Russo IH, Koszalka M \& Russo J 1990 Human chorionic gonadotropin and rat mammary cancer prevention. Journal of the National Cancer Institute 82 1286-1289.

Russo IH, Koszalka M \& Russo J 1991 Comparative study of the influence of pregnancy and hormonal treatment on mammary carcinogenesis. British Journal of Cancer 64 481-484.

Russo J, Rivera R \& Russo IH 1992 Influence of age and parity on the development of the human breast. Breast Cancer Research and Treatment 23 211-218.

Russo J, Ao X, Grill C \& Russo IH 1999 Pattern of distribution of cells positive for estrogen receptor $\alpha$ and progesterone receptor in relation to proliferating cells in the mammary gland. Breast Cancer Research and Treatment 53 217-227.

Russo J, Mailo D, Hu Y-F, Balogh G, Sheriff F \& Russo IH 2005 Breast differentiation and its implication in cancer prevention. Clinical Cancer Research 11 931S-936S.

Santen RJ 2003 Risk of breast cancer with progestins: critical assessment of current data. Steroids 68 953-964.

Schedin P, Mitrenga T, McDaniel S \& Kaeck M 2004 Mammary ECM composition and function are altered by reproductive state. Molecular Carcinogenesis 41 207-220.

Sinha DK, Pazik JE \& Dao TL 1988 Prevention of mammary carcinogenesis in rats by pregnancy: Effect of full-term and interrupted pregnancy. British Journal of Cancer 57 390-394.

Sivaraman L, Stephens LC, Markaverich BM, Clark JA, Krnacik S, Conneely OM, O'Malley BW \& Medina D 1998 Hormone-induced refractoriness to mammary carcinogenesis in Wistar-Furth rats. Carcinogenesis 19 1573-1581.

Sivaraman L, Conneely OM, Medina D \& O'Malley BW $2001 a$ p53 is a potential mediator of pregnancy and hormone-induced resistance to mammary carcinogenesis. PNAS 98 12379-12384.

Sivaraman L, Hilsenbeck SG, Zhong L, Gay J, Conneely OM, Medina D \& O'Malley BW $2001 b$ Early exposure of the rat mammary gland to estrogen and progesterone blocks colocalization of ER expression and proliferation. Journal of Endocrinology 170 75-83. 
Srivastava P, Russo J \& Russo IH 1997 Chorionic gonadotropin inhibits rat mammary carcinogenesis through activation of programmed cell death. Carcinogenesis 18 1799-1908.

Swanson SM, Guzman RC, Collins G, Tafoya P, Thordarson G, Talamantes F \& Nandi S 1995 Refractoriness to mammary carcinogenesis in the parous mouse is reversible by hormonal stimulation induced by pituitary isografts. Cancer Letters 90 171-181.

Thordarson G, Jin E, Guzman RC, Swanson SM, Nandi S \& Talamantes F 1995 Refractoriness to mammary tumorigenesis in parous rats: Is it caused by persistent changes in the hormonal environment or permanent biochemical alterations in the mammary epithelia? Carcinogenesis 16 2847-2853.

Thordarson G, Van Horn K, Guzman RC, Nandi S \& Talamantes F 2001 Parous rats regain high susceptibility to chemically induced mammary cancer after treatment with various mammotropic hormones. Carcinogenesis 22 1027-1033.

Thordarson G, Semaan S, Low C, Ochoa D, Leong H, Rajkumar L, Guzman RC, Nandi S \& Talamantes F 2004 a Mammary tumorigenesis in growth hormone deficient spontaneous dwarf rats; effects of hormonal treatments. Breast Cancer Research and Treatment $\mathbf{8 7}$ 277-290.

Thordarson G, Slusher N, Leong H, Ochoa D, Rajkumar L, Guzman R, Nandi S \& Talamantes F 2004b Insulin-like growth factor (IGF)-I obliterates the pregnancyassociated protection against mammary carcinogenesis in rats: evidence that IGF-I enhances cancer progression through estrogen receptor-alpha activation via the mitogen-activated protein kinase pathway. Breast Cancer Research 6 R423-R436.
Tokunaga M, Land C, Tokuoka S, Nishimori I, Soda M \& Akiba S 1994 Incidence of female breast cancer among atomic bomb survivors. Radiation Research 138 209-223.

Ursin G, Bernstein L, Wang Y, Lord SJ, Deapen D, Liff JM, Norman SA, Weiss LK, Daling JR, Marchbanks PA et al. 2004 Reproductive factors and risk of breast carcinoma in a study of white and African-American women. Cancer 101 353-362.

Wagner KU, Boulanger CA, Henry MD, Sgagias M, Hennighausen L \& Smith GH 2002 An adjunct mammary epithelial cell population in parous females: its role in functional adaptation and tissue renewal. Development 129 1377-1386.

Wattenberg LW 1997 An overview of chemoprevention: current status and future projects. Proceedings of Experimental Biology and Medicine 216 133-141.

Welsch CW 1987 Dietary Retinoids and the Chemoprevention of Mammary Gland Morphogenesis. In Cell and Molecular Biology of Experimental Breast Cancer, pp 495-508. Eds D Medina, GH Hepper, WR Kidwell \& E Anderson. New York: Plenum Press.

Wu AH, Wan P, Hankin J, Tseng CC, Yu MC \& Pike MC 2002 Adolescent and adult soy intake and risk of breast cancer in Asian-Americans. Carcinogenesis 23 1491-1496.

Wu K, Zhang Y, Xu HC, Hill J, Celestino J, Kim HT, Mohsin SK, Hilsenbeck SG, Lamph WW, Bissonette R \& Brown PH 2002 The retinoid X receptor-selective retinoid, LGD1069, prevents the development of estrogen receptor-negative mammary tumors in transgenic mice. Cancer Research 62 6376-6380.

Yang J, Yoshizawa K, Nandi S \& Tsubura A 1999 Protective effects of pregnancy and lactation against $N$-methyl- $N$ nitrosourea-induced mammary carcinomas in female Lewis rats. Carcinogenesis 20 623-628. 
